\title{
Assessment of Causal Relationship Between Rheumatoid Arthritis and Inflammatory Bowel Disease: A Bi-Directional Mendelian Randomization Study
}

\author{
Xueer Zhang \\ Peking University \\ Zhenhuang Zhuang \\ Peking University \\ Jie Du \\ Peking University \\ Wenxiu Wang \\ Peking university \\ Ruotong Yang \\ Peking University \\ Zhonghua Liu
}

The university of HongKong

Tao Huang ( $\nabla$ huangtaotao@pku.edu.cn )

Peking University https://orcid.org/0000-0002-5774-7493

Research article

Keywords: Rheumatoid arthritis, Inflammatory bowel disease, Mendelian Randomization

Posted Date: October 22nd, 2020

DOI: https://doi.org/10.21203/rs.3.rs-93718/v1

License: (c) (1) This work is licensed under a Creative Commons Attribution 4.0 International License. Read Full License 


\section{Abstract}

Objective: Observational studies have shown that rheumatoid arthritis (RA) was associated with a higher risk of inflammatory bowel disease (IBD), and vice versa. However, if such associations reflect causality remains unclear. We aimed to examine the bidirectional causal associations of RA with IBD, ulcerative colitis (UC), and Crohn's disease (CD).

Method: A Bi-directional Mendelian randomization (MR) analysis and Linkage Disequilibrium Score regression (LDSC) were used to investigate the causality between RA and IBD subtypes. Summary data was extracted from the Rheumatoid Arthritis Consortium International for Immunochip (RACI) consortium and the Genetics and Allied research in Rheumatic diseases Networking (GARNET) consortium $(29,880$ cases of RA and 73,758 controls); and the International IBD Genetics Consortium (38,155 cases of IBD and 48,485 controls; 17,647 cases of UC and 47,179 controls; 20,550 cases of CD and 41,642 controls).

Results: Genetically predicted RA was associated with higher risks of IBD (per 1-unit higher log odds: odds ratio: 1.13; $95 \%$ confidence interval: $1.04-1.21 ; \mathrm{P}=0.002)$ and UC $\left(1.16 ; 1.07-1.25 ; \mathrm{P}=1.5 \times 10^{-4}\right)$ after a Bonferroni correction $(P<0.05 / 3)$. In addition, the weighted median method showed a suggestive association of RA with a higher risk of $C D(1.16 ; 1.05-1.28 ; P=0.003)$. However, there was no evidence showing a causal relation of IBD, UC or CD with RA risk.

Conclusion: Our findings provide novel evidence supporting a significant causality of RA with IBD or UC, whereas IBD is unlikely to increase the risk of RA, indicating the importance of keeping gut microbiota composition healthy for RA patients to prevent IBD.

\section{Introduction}

Rheumatoid arthritis (RA) and inflammatory bowel disease (IBD) are two inflammatory diseases involved with innate and adaptive immune components that change the metabolic state of their respective target tissues. ${ }^{1}$ Accumulating evidence has suggested that an abnormal overall composition of intestinal microbiota plays an important role in the pathogenesis of RA and IBD, 2,3 indicating overlaps between pathogenic pathways. Therefore, figuring out the bidirectional causal relationship between RA and IBD may facilitate early diagnosis and prevention of complications.

Compelling evidence from observational studies such as case-control studies ${ }^{4-6}$ and large crosssectional study ${ }^{7}$ has consistently shown that RA is significantly associated with a higher risk of IBD, ulcerative colitis (UC) and Crohn's disease (CD). In fact, these observational studies largely relying on selfreported information are susceptible to measured or unmeasured confounders and reverse causation bias, thus difficult to infer causality among RA and IBD, UC and CD.

Mendelian Randomization (MR) analysis is a sort of natural randomized control trial method at the genetic level, when randomization occurs during meiosis and conception, and genotype is hardly likely to be affected after birth. ${ }^{8}$ Thus, genetic instrumental variables (IVs) are relatively independent from any 
environmental factors or other developed diseases, ensuring that MR method can largely avoid confounders and reverse causation bias using IVs. ${ }^{9}$ To our knowledge, no MR analysis of the association between RA and IBD thus far have been conducted.

Hence, in the present study, we for the first time performed a bi-directional MR analysis using summerylevel statistics from large genome-wide association study (GWAS) consortium to assess the bidirectional causal associations between RA and IBD, UC or CD.

\section{Methods}

\section{Research Structure}

To eliminate the interference of confounders and reverse causation, we performed a bi-directional MR analysis by teasing apart the causal effect of RA on the risks of IBD, UC and CD and the reverse causal effect, respectively. The MR analysis was first conducted in one direction (RA to IBD, UC and CD) and then conducted in the opposite direction (IBD, UC and CD to RA) with the genetic variants robustly associated with each disease in the separate GWAS. The MR approach should be based on three core assumptions: genetic variants used as IVs are associated with the exposure (assumption 1); genetic variants are not associated with any confounders (assumption 2); genetic variants influence the risk of outcome only through exposure, not through any alternative pathways (assumption 3) (Figure 1).

\section{Data Sources of RA}

Summary statistics for RA were extracted from a genome-wide association study (GWAS) meta-analysis in a total of $>100,000$ subjects of European and Asian ancestries (29,880 cases and 73,758 controls). ${ }^{10}$ This GWAS meta-analysis based on European and Asian ancestries contains data from two consortia including the Rheumatoid Arthritis Consortium International for Immunochip (RACl) consortium and the Genetics and Allied research in Rheumatic diseases Networking (GARNET) consortium(Table 1). All RA cases fulfilled the 1987 criteria of the American College of Rheumatology or were diagnosed by a professional rheumatologist. The GWAS identified 102 single nucleotide polymorphisms (SNPs) genomewide significantly associated with RA (identified as $\mathrm{P}<5 \times 10^{-8}$ ). However, 11 of the RA-related SNPs was unavailable in the International IBD Genetics Consortium, leaving only 91 SNPs as IVs. The details of instrumental SNPs could be seen in eTable 1.

\section{Data Sources of IBD, UC and CD}

Summary-level data were extracted from a recent GWAS replication and meta-analysis based on the European GWAS and Immunochip in the International IBD Genetics Consortium, involving 213,658 participants including 38,155 IBD cases and 48,485 controls, 17,647 UC cases and 47,179 controls, and 20,550 CD cases and 41,642 controls (Table 1). ${ }^{11}$ The diagnosis of IBD was based on the accepted clinical criteria of radiologic, endoscopic, and histopathologic evaluation. This GWAS meta-analysis identified 27 SNPs for IBD, 4 SNPs for UC and 7 SNPs for CD (identified as $P<5 \times 10^{-8}$ in European 
ancestry or $\log 10$ Bayes factor $>6$ in the combined trans-ancestry association analysis) and 2 SNPs associated with IBD was unavailable in the RACl and GARNET consortium, leaving 25 SNPs for IBD, 4 SNPs for UC and 7 SNPs for CD is as IVs (eTable 3).

\section{Statistical Analysis}

\section{Assessment of Linkage Disequilibrium}

To meet assumption 1 and 2, the IV must be associated with RA or IBD, UC and CD, and not with other confounders. We assessed the linkage disequilibrium (LD) correlation among SNPs of IBD in order to select independent genetic variants $\left(r^{2}<0.1\right)$. We chose the one with the lowest $P$ value associated with $I B D, U C$ and $C D$, if genetic variants are in $L D\left(r^{2}>0.8\right)$. $F$ statistic was used to test the strength of genetic variants, and $\mathrm{F}>10$ was identified as meeting assumption 1 .

\section{Mendelian Randomization and sensitivity analysis}

We used the inverse variance weighted method in the MR analysis as the main analysis method, which presents a combined estimate of the causal estimate from each SNP. ${ }^{12}$ To conduct MR analysis with this method, we had to assume that there was no SNP had horizontal pleiotropy. Then we performed sensitivity analyses for the IVs significantly related to exposure with the simple median, weighted median and MR Egger regression methods. Given that not all IVs were valid, the simple median was used to provide with a consistent effect estimate when at least $50 \%$ of IVs were valid. ${ }^{13}$ Compared to the simple median, the weighted median could provide a consistent effect estimate when at least $50 \%$ of the weight was from valid IVs, presenting the robustness in the causal estimate of the exposure-outcome effect. ${ }^{13}$ As for MR Egger regression method, the zero intercept result was considered nearly no pleiotropy effect; ${ }^{14}$ thus showing the pleiotropy-corrected effect. ${ }^{15}$ To deeper examine the effect of influential or pleiotropy variants ,we performed leave-one-out analysis, where one variant was neglected at one time. ${ }^{16}$ Power calculations in the MR analysis were conducted based on the website: $\mathrm{mRnd}$ (http://cnsgenomics.com/shiny/mRnd/).

All analyses above were performed in the R version 3.6.1 computing environment (http://www.rproject.org) using the TwoSampleMR package (R project for Statistical Computing). This package harmonized effect of exposure and outcome data sets including combined information on SNPs, effect alleles, non-effect alleles, effect estimates, standard errors for instrumental SNPs. By the way, we assumed that all alleles are presented on the forward strand in harmonization. Therefore, the bidirectional results took the full set of instrumental SNPs into account. In addition, we applied a conservative approach considering multiple testing and set a significant threshold as 0.017 (0.05 divided by 3) after Bonferroni's correction. $P \leq 0.05$ but above the Bonferroni corrected significance threshold was considered as a suggestive significant association.

\section{LD Score regression}


To evaluate the genetic correlation between RA and IBD, UC or CD, we performed LDSC by using GWAS summary statistics from two previous trait-specific GWASs. LDSC estimated genetic correlations between the true causal effects of RA and IBD, UC or CD (ranging from -1 to 1) using GWAS summary statistics of Hapmap3 SNPs. SNPs with high LD region would have higher $\chi^{2}$ statistics than SNPs with a lower LD region for each disease, and a similar relationship held if single-study test statistics were replaced with the product of the $z$ scores from two studies of traits with some correlation. ${ }^{17}$

\section{Results}

\section{Characteristics of instrumental variables}

Table 1 shows the sample size, population and publication year of GWASs for RA, IBD, UC and CD. We discarded variants from the association analysis which were not available and with no proxy in the outcome GWASs. We tested whether the selected SNPs were affected by LD, and ultimately chose the variant with the lowest $P$ value if genetic variants are in LD. Finally, we identified 91 SNPs for RA, 25 SNPs for IBD, 4 SNPs for UC, 7 SNPs for CD (eTable 1 and eTable 3). Characteristics of selected SNPs including effect allele, effect allele frequency, effect estimate, standard error and other available information in are list in eTable 1-4. Furthermore, we found that all SNPs for the same trait show strong strength (Fstatistic $>10)$. We used MR power calculation to detect the significant $(P=0.05 / 3)$ causal effect $(O R=1.3)$ of RA on IBD, UC and CD, and found that the powers were strong enough (more that $80 \%$ ). In the other direction, this MR power calculation showed that we have $98 \%$ power to test the significant $(P=0.05 / 3)$ causal relationship $(\mathrm{OR}=1.3)$ of IBD on RA. However, when detecting the causal relationship of UC or CD on RA, we found the powers were both less than $80 \%$.

\section{Causal effect of RA on IBD, UC or CD}

We found that genetically predicted higher RA was significantly associated with higher odds of IBD and UC. Per unit higher log odds of RA, the odds ratio (OR) of IBD were 1.13 (95\% confidence interval $(\mathrm{Cl})$, 1.04-1.21; $P=0.002)$ and $1.16\left(1.07-1.25 ; P=1.5 \times 10^{-4}\right)$ of UC (Figure 2). Furthermore, the sensitivity analyses using the simple median (1.12 [1.04-1.21; $P=0.002]$ of IBD and 1.13 [1.05-1.22; $P=0.002]$ of UC) and the weighed median (1.10 [1.02-1.18; $P=0.014]$ of IBD and 1.12 [1.02-1.24; $P=0.018]$ of UC) confirmed the associations (Figure 2). The intercepts of MR Egger regression method for each outcome, which were centered at the origin with a $\mathrm{Cl}$ including the null, further suggested no horizontal pleiotropy inflated effect between those variants. (Figure 2). Regarding $C D$, the weighted median method $(1.16 ; 1.05-1.28 ; P=0.003$ ) and the simple median method $(1.18 ; 1.07-1.30 ; P=0.001)$ indicated a causal association of RA with CD (Figure 2). However, the IVW method $(1.08 ; 0.97-1.20 ; \mathrm{P}=0.154)$ showed a similar trend, but not a significant association (Figure 2). In the leave-one-out analysis, we did not identify any influential variants or outlier in the associations of RA with IBD or UC (eTable 5). As for SNP effect size upon IBD and UC versus that upon RA, we plotted two graphs to present the estimated results through different MR methods (eFigure 1 and eFigure 2). Moreover, MR estimate of IVs versus instrument precision was also plotted to examine evidence of directional pleiotropy (eFigure 1 and eFigure 2). 


\section{Causal effect of IBD, UC or CD on RA}

In the reverse-direction MR analysis, genetically predicted higher IBD, UC and CD was not associated with the risk of RA. The OR were $0.86(0.72-1.03 ; P=0.10)$ per unit higher log odds of IBD, 0.91 (0.74-1.12; $\mathrm{P}=0.37)$ per unit higher log odds of $U C$, and $1.12(0.88-1.43 ; \mathrm{P}=0.37)$ per unit higher log odds of $C D$ (Figure 3). Likewise, in the sensitivity analyses, both the weighted median method and the simple median method showed the similar results for IBD, UC and CD (Figure 3). In addition, the leave-one-out analysis results showed that the $O R$ ranged from $0.83(0.70-0.98 ; P=0.029)$ when we excluded the genetic variant (rs 4703855) to $0.83(0.70-0.99 ; \mathrm{P}=0.036)$ when we excluded the variant (rs 4692386) (eTable 6).

\section{Discussion}

In the present study, we systematically assessed the causal relationships and genetic correlation between RA and IBD subtypes. We found strong evidence supporting causal associations of genetically predicted RA and higher risks of IBD and UC and significant genetic correlation of RA and UC, whereas IBD is unlikely to increase the risk of RA, suggesting RA patients may have higher risk of developing IBD. Our findings indicated that keeping an optimal healthy gut microbiota composition might have impressive clinical benefits for RA patients to prevent the development of IBD.

Growing evidence from observational studies has suggested that RA is associated with a higher risk of IBD. ${ }^{3,7}$ For example, IBD was observed with greater frequency among patients with RA in a previous observational study $(n=63,005) .{ }^{7}$ Our results were also in line with the evidence from a recent small cohort study $(n=102)$ presenting a potential links to gut microbiota composition between RA and UC. ${ }^{18}$ Furthermore, a large cross-sectional study with a total of 47,325 IBD cases and 92,839 healthy controls suggested that IBD patients tended to have co-occurring RA problems. ${ }^{3}$ However, Vanessa et al ${ }^{6}$ conducted a case-control study $(n=3,284)$ showing that IBD was associated with RA at any time but even more so in the period before RA diagnosis. In addition, another case-control study ( $n=4,532)$ using paediatric-specific data to evaluate the prevalence of RA among children with IBD showed no significant association between RA and UC, but observe a strong association between RA and CD. ${ }^{19}$ In fact, these small observational studies (sample size ranging from 102 to 4,532) are difficult to interpret due to small sample size and bias from unmeasured confounding factors, short-term effect and/or reverse causation. Our bi-directional MR study, for the first time, provided strong evidence for the bidirectional causal associations between RA and IBD, UC and CD as well as revealed the genetic basis of RA and IBD by leveraging summery-level statistics from large GWAS consortium which was in line with an association direction observed from epidemiological studies above, whereas IBD is unlikely to increase the risk of RA.

Many biologically plausible pathways linking RA to a higher risk of IBD have been identified. One possible pathway is through gut dysbiosis which occurs in the inflammatory process and plays a crucial role in the pathogenesis and development of both RA and IBD. ${ }^{2,20-24}$ The alterations of intestinal microbiota composition in RA have a considerable overlap with those in IBD, ${ }^{25,26}$ among which some bacterial taxa, such as Pseudomonas, have showed consistent trends of changes. ${ }^{27}$ Interestingly, a comparative study 
suggested that the gut microbial communities in RA and UC were most similar among immune-mediated inflammatory diseases, while the gut microbiota of $C D$ was most different from other immune-mediated inflammatory diseases. ${ }^{18}$ Taken together, these findings might at least partially support our results. In addition, previous studies revealed that gut flora might be involved in the development of RA and immune disorders by activating Th17 cell. ${ }^{28-30}$ Correspondingly, the role of intestinal microbiota-reactive Th17 cells is also identified as mediating the pathogenesis of IBD, ${ }^{31}$ further confirming the potential causal pathway linking RA to IBD. However, further investigations are required to elucidate the underlying mechanism of gut microbiota-host crosstalk in both RA and IBD.

There are several strengths in our study. To the best of our knowledge, we for the first time assessed the causal relationship between RA and IBD using summary-level data from large GWAS, which are not generally susceptible to reverse causation and confounding. Importantly, the results from four different MR methods are consistent, showing the robustness of this study. In addition, the power calculated from summary-level data was sufficient, thus proving the reliable causal estimation. However, several limitations merit consideration. First, although we have exploited the large genome-wide summary-level data and carefully selected SNPs, the possibility of potential weak instrument bias couldn't be ruled out. To address this, F-statistic can reliably detect the strength of $\mathrm{IVs}^{32}$ which is a proof of our strong instrument strength. Second, it is a great challenge for all MR analysis to exclude pleiotropy and other alternative direct causal pathway, especially for those diseases determined by genetic variants, ${ }^{33}$ although we applied additional sensitivity analyses to validate the robustness of our results. Finally, the association between RA and UC could not distinguish share genetic basis from causal relationship because human genome influenced both RA and IBD.

In summary, our MR analysis provides strong evidence supporting associations direction from genetically predicted RA to higher risks of IBD and UC, suggesting RA patients might have intestinal microbiome variation and higher risk of developing IBD. However, IBD is unlikely to increase risk of RA. Our findings have clinical significance for preventing IBD for patients with RA, and underscore the importance of maintaining an optimal healthy intestinal microbial population composition. Further studies assessing the clinical significance of intestinal microbiome variation in RA patients are required.

\section{Declarations}

\section{Contributions}

$\mathrm{XZ}, \mathrm{ZZ}$ and TH designed the research. $\mathrm{XZ}$ and TH had full access to all the data in the study and take responsibility for the integrity of the data and the accuracy of the data analysis. $\mathrm{XZ}, \mathrm{ZZ}$ and $\mathrm{TH}$ wrote the paper and performed the data analysis. All authors contributed to the statistical analysis, critically reviewed the manuscript during the writing process, and approved the final version to be published. XZ and $\mathrm{TH}$ are the guarantors for the study.

\section{Data Availability.}


All data used in the present study were obtained from genome wide association study summary statistics which were publicly released by genetic consortia.

\section{Ethical approval and consent to participate}

Contributing studies received ethical approval from their respective institutional review boards. Informed consent was obtained from all participants of contributing studies.

\section{Funding}

The study was supported by grants from the Peking University Start-up Grant (BMU2018YJ002), Highperformance Computing Platform of Peking University. The funding organization had no role in the preparation of the manuscript.

\section{Disclosure Statement}

All authors declare: no support from companies for the submitted work; no relationships with companies that might have an interest in the submitted work in the previous three years; no spouses, partners, or children have no financial relationships that may be relevant to the submitted work; no non-financial interests that may be relevant to the submitted work.

\section{References}

1. McBride HJ. Nuclear imaging of autoimmunity: focus on IBD and RA. Autoimmunity. 2010;43(7):539549.

2. Nikitakis NG, Papaioannou W, Sakkas LI, Kousvelari E. The autoimmunity-oral microbiome connection. Oral diseases. 2017;23(7):828-839.

3. Halling ML, Kjeldsen J, Knudsen T, Nielsen J, Hansen LK. Patients with inflammatory bowel disease have increased risk of autoimmune and inflammatory diseases. World journal of gastroenterology. 2017;23(33):6137-6146.

4. Bae JM, Choo JY, Kim KJ, Park KS. Association of inflammatory bowel disease with ankylosing spondylitis and rheumatoid arthritis: A nationwide population-based study. Modern rheumatology. 2017;27(3):435-440.

5. Cohen R, Robinson D, Jr., Paramore C, Fraeman K, Renahan K, Bala M. Autoimmune disease concomitance among inflammatory bowel disease patients in the United States, 2001-2002. Inflammatory bowel diseases. 2008;14(6):738-743.

6. Kronzer VL, Crowson CS, Sparks JA, Myasoedova E, Davis JM, 3rd. Comorbidities As Risk Factors for Rheumatoid Arthritis and Their Accrual After Diagnosis. Mayo Clin Proc. 2019;94(12):2488-2498.

7. Weng X, Liu L, Barcellos LF, Allison JE, Herrinton LJ. Clustering of inflammatory bowel disease with immune mediated diseases among members of a northern california-managed care organization. The American journal of gastroenterology. 2007;102(7):1429-1435. 
8. Sekula P, Del Greco MF, Pattaro C, Kottgen A. Mendelian Randomization as an Approach to Assess Causality Using Observational Data. Journal of the American Society of Nephrology : JASN. 2016;27(11):3253-3265.

9. Emdin CA, Khera AV, Kathiresan S. Mendelian Randomization. Jama. 2017;318(19):1925-1926.

10. Okada Y, Wu D, Trynka G, et al. Genetics of rheumatoid arthritis contributes to biology and drug discovery. Nature. 2014;506(7488):376-381.

11. Liu JZ, van Sommeren S, Huang H, et al. Association analyses identify 38 susceptibility loci for inflammatory bowel disease and highlight shared genetic risk across populations. Nature genetics. 2015;47(9):979-986.

12. Geng T, Smith CE, Li C, Huang T. Childhood BMI and Adult Type 2 Diabetes, Coronary Artery Diseases, Chronic Kidney Disease, and Cardiometabolic Traits: A Mendelian Randomization Analysis. Diabetes care. 2018;41(5):1089-1096.

13. Bowden J, Davey Smith G, Haycock PC, Burgess S. Consistent Estimation in Mendelian Randomization with Some Invalid Instruments Using a Weighted Median Estimator. Genetic epidemiology. 2016;40(4):304-314.

14. Noyce AJ, Kia DA, Hemani G, et al. Estimating the causal influence of body mass index on risk of Parkinson disease: A Mendelian randomisation study. PLoS medicine. 2017;14(6):e1002314.

15. Bowden J, Davey Smith G, Burgess S. Mendelian randomization with invalid instruments: effect estimation and bias detection through Egger regression. International journal of epidemiology. 2015;44(2):512-525.

16. Burgess S, Thompson SG. Interpreting findings from Mendelian randomization using the MR-Egger method. European journal of epidemiology. 2017;32(5):377-389.

17. Bulik-Sullivan B, Finucane HK, Anttila V, et al. An atlas of genetic correlations across human diseases and traits. Nature genetics. 2015;47(11):1236-1241.

18. Forbes JD, Chen C-Y, Knox NC, et al. A comparative study of the gut microbiota in immune-mediated inflammatory diseases-does a common dysbiosis exist? Microbiome. 2018;6(1):221-221.

19. Kappelman MD, Galanko JA, Porter CQ, Sandler RS. Association of paediatric inflammatory bowel disease with other immune-mediated diseases. Archives of disease in childhood. 2011;96(11):10421046.

20. Lin L, Zhang J. Role of intestinal microbiota and metabolites on gut homeostasis and human diseases. BMC Immunol. 2017;18(1):2-2.

21. Muszer M, Noszczyńska M, Kasperkiewicz K, Skurnik M. Human Microbiome: When a Friend Becomes an Enemy. Arch Immunol Ther Exp (Warsz). 2015;63(4):287-298.

22. Picchianti-Diamanti A, Panebianco C, Salemi S, et al. Analysis of Gut Microbiota in Rheumatoid Arthritis Patients: Disease-Related Dysbiosis and Modifications Induced by Etanercept. Int J Mol Sci. 2018;19(10):2938. 
23. Zhang Y-Z, Li Y-Y. Inflammatory bowel disease: pathogenesis. World journal of gastroenterology. 2014;20(1):91-99.

24. Barko PC, McMichael MA, Swanson KS, Williams DA. The Gastrointestinal Microbiome: A Review. J Vet Intern Med. 2018;32(1):9-25.

25. Breban M. Gut microbiota and inflammatory joint diseases. Joint Bone Spine. 2016;83(6):645-649.

26. Yeoh N, Burton JP, Suppiah P, Reid G, Stebbings S. The role of the microbiome in rheumatic diseases. Curr Rheumatol Rep. 2013;15(3):314-314.

27. Salem F, Kindt N, Marchesi JR, et al. Gut microbiome in chronic rheumatic and inflammatory bowel diseases: Similarities and differences. United European Gastroenterol J. 2019;7(8):1008-1032.

28. Hasler P, Gabay C. Rheumatoid arthritis: from basic findings and clinical manifestations to future therapies. Semin Immunopathol. 2017;39(4):339-341.

29. Kim D, Zeng MY, Núñez G. The interplay between host immune cells and gut microbiota in chronic inflammatory diseases. Exp Mol Med. 2017;49(5):e339-e339.

30. Kochetkova I, Trunkle T, Callis G, Pascual DW. Vaccination without autoantigen protects against collagen II-induced arthritis via immune deviation and regulatory T cells. Journal of immunology (Baltimore, Md : 1950). 2008;181(4):2741-2752.

31. Wu W, Chen F, Liu Z, Cong Y. Microbiota-specific Th17 Cells: Yin and Yang in Regulation of Inflammatory Bowel Disease. Inflammatory bowel diseases. 2016;22(6):1473-1482.

32. Burgess S, Thompson SG. Bias in causal estimates from Mendelian randomization studies with weak instruments. Stat Med. 2011;30(11):1312-1323.

33. Larsson SC, Traylor M, Malik R, et al. Modifiable pathways in Alzheimer's disease: Mendelian randomisation analysis. BMJ (Clinical research ed). 2017;359:j5375-j5375.

\section{Tables}


Table 1

Description of GWAS or consortium for RA, IBD, UC and CD.

\begin{tabular}{|llllll|}
\hline Disease & Consortium or study & Sample size & Populations & Journal & Year \\
\hline RA & $\begin{array}{l}\text { the RACl and GARNET } \\
\text { consortium }\end{array}$ & $\begin{array}{l}29,880 \text { cases and } \\
73,758 \text { controls }\end{array}$ & $\begin{array}{l}\text { Trans-ethnic } \\
\text { European Asian }\end{array}$ & Nature & 2014 \\
\hline IBD & $\begin{array}{l}\text { the International IBD } \\
\text { Genetics Consortium }\end{array}$ & $\begin{array}{l}38,155 \text { cases and } \\
48,485 \text { controls }\end{array}$ & European & $\begin{array}{l}\text { Nat } \\
\text { Genet }\end{array}$ & 2015 \\
\hline UC & $\begin{array}{l}17,647 \text { cases and } \\
\text { the International IBD } \\
\text { Genetics Consortium }\end{array}$ & $\begin{array}{l}\text { European } \\
\text { CD }\end{array}$ & $\begin{array}{l}\text { Nat } \\
\text { Genet }\end{array}$ & 2015 \\
\hline & $\begin{array}{l}\text { the International IBD } \\
\text { Genetics Consortium }\end{array}$ & $\begin{array}{l}20,550 \text { cases and } \\
41,642 \text { controls }\end{array}$ & European & $\begin{array}{l}\text { Nat } \\
\text { Genet }\end{array}$ & 2015 \\
\hline $\begin{array}{l}\text { Abbreviations: RACl, Rheumatoid Arthritis Consortium International for Immunochip; GARNET, } \\
\text { Genetics and Allied research in Rheumatic diseases Networking; RA, rheumatoid arthritis; IBD, } \\
\text { inflammatory bowel disease; UC, ulcerative colitis; CD, Crohn's disease. }\end{array}$ \\
\hline
\end{tabular}

\section{Figures}


Instrumental variables(IVs)

Polygenic risk score for RA

or IBD, UC and CD
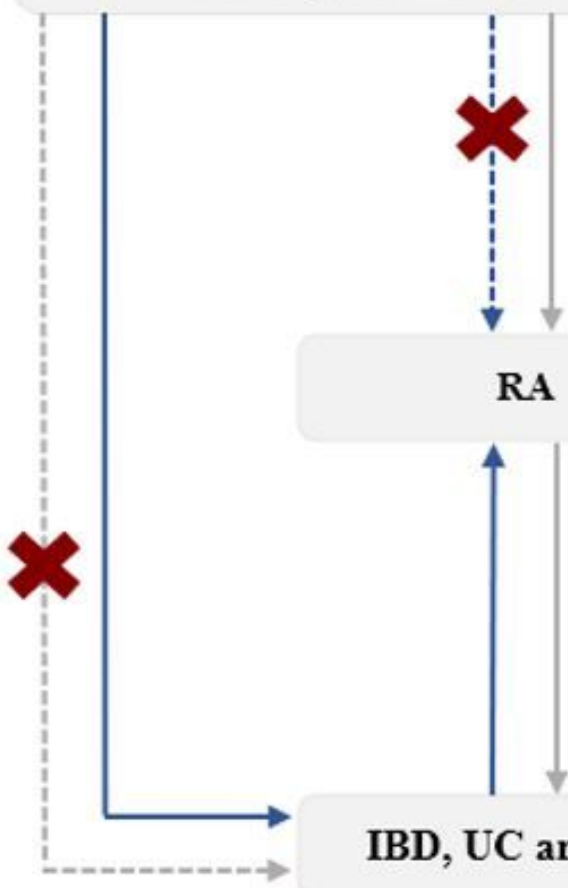

RA

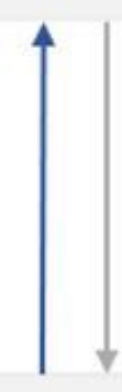

IBD, UC and CD

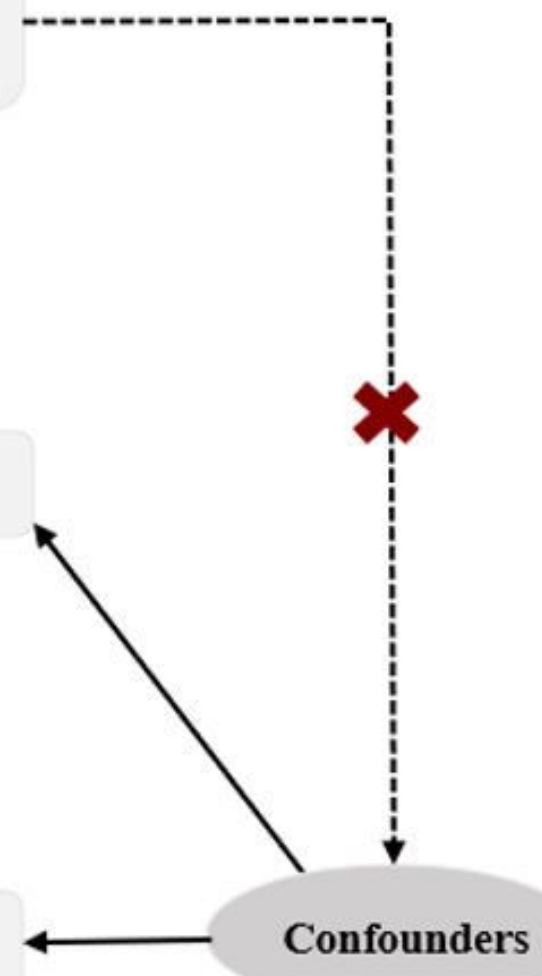

Confounders

\section{Figure 1}

Mendelian Randomization Model. Three assumptions of MR: (1) Genetic variants are associated with the exposure; (2) Genetic variants are not associated with any confounders; (3) Genetic variants influence the risk of outcome only through exposure, not through any alternative pathways. Gray lines represent the MR analysis on causal effects of RA on IBD, UC and CD, while blue lines represent the analysis in the opposite direction. RA, rheumatoid arthritis; IBD, inflammatory bowel disease; UC, ulcerative colitis; CD, Crohn's disease. 


\begin{tabular}{|c|c|c|c|c|}
\hline Disease & No. of SNPs & & OR(95\%Cl) & P-value \\
\hline \multicolumn{5}{|l|}{ IBD } \\
\hline Simple median & 91 & - & $1.12(1.04,1.21)$ & 0.002 \\
\hline Weighted median & 91 & $\rightarrow-$ & $1.10(1.02,1.18)$ & 0.014 \\
\hline Inverse variance weighted & 91 & - & $1.13(1.04,1.21)$ & 0.002 \\
\hline MR Egger & 91 & $=$ & $0.99(0.84,1.17)$ & 0.918 \\
\hline (Intercept) & 91 & = & $1.02(1.00,1.04)$ & 0.100 \\
\hline \multicolumn{5}{|l|}{ UC } \\
\hline Simple median & 91 & $\rightarrow-$ & $1.13(1.05,1.22)$ & 0.002 \\
\hline Weighted median & 91 & $\rightarrow-$ & $1.12(1.02,1.24)$ & 0.018 \\
\hline Inverse variance weighted & 91 & $\rightarrow-$ & $1.16(1.07,1.25)$ & $1.5 \times 10^{-4}$ \\
\hline MR Egger & 91 & $=$ & $1.13(0.95,1.33)$ & 0.178 \\
\hline (Intercept) & 91 & - & $1.00(0.98,1.02)$ & 0.708 \\
\hline \multicolumn{5}{|l|}{ CD } \\
\hline Simple median & 91 & $\rightarrow-$ & $1.18(1.07,1.30)$ & 0.001 \\
\hline Weighted median & 91 & $\rightarrow-$ & $1.16(1.05,1.28)$ & 0.003 \\
\hline Inverse variance weighted & 91 & $\rightarrow-$ & $1.08(0.97,1.20)$ & 0.154 \\
\hline MR Egger & 91 & - & $0.86(0.67,1.09)$ & 0.212 \\
\hline \multirow[t]{2}{*}{ (Intercept) } & 91 & $=$ & $1.03(1.00,1.06)$ & 0.040 \\
\hline & 0.5 & $\begin{array}{l}1 \\
1\end{array}$ & 5 & \\
\hline
\end{tabular}

\section{Figure 2}

Odds ratio for association of genetically predicted RA with IBD, UC and CD, using four different Mendelian randomization methods. OR: odds ratio; $\mathrm{Cl}$ : confidence internal; RA, rheumatoid arthritis; IBD, inflammatory bowel disease; UC, ulcerative colitis; CD, Crohn's disease. OR (95\% Cl) means risk of IBD, UC and CD per each 1-unit higher log odds in genetically predicted rheumatoid arthritis. 


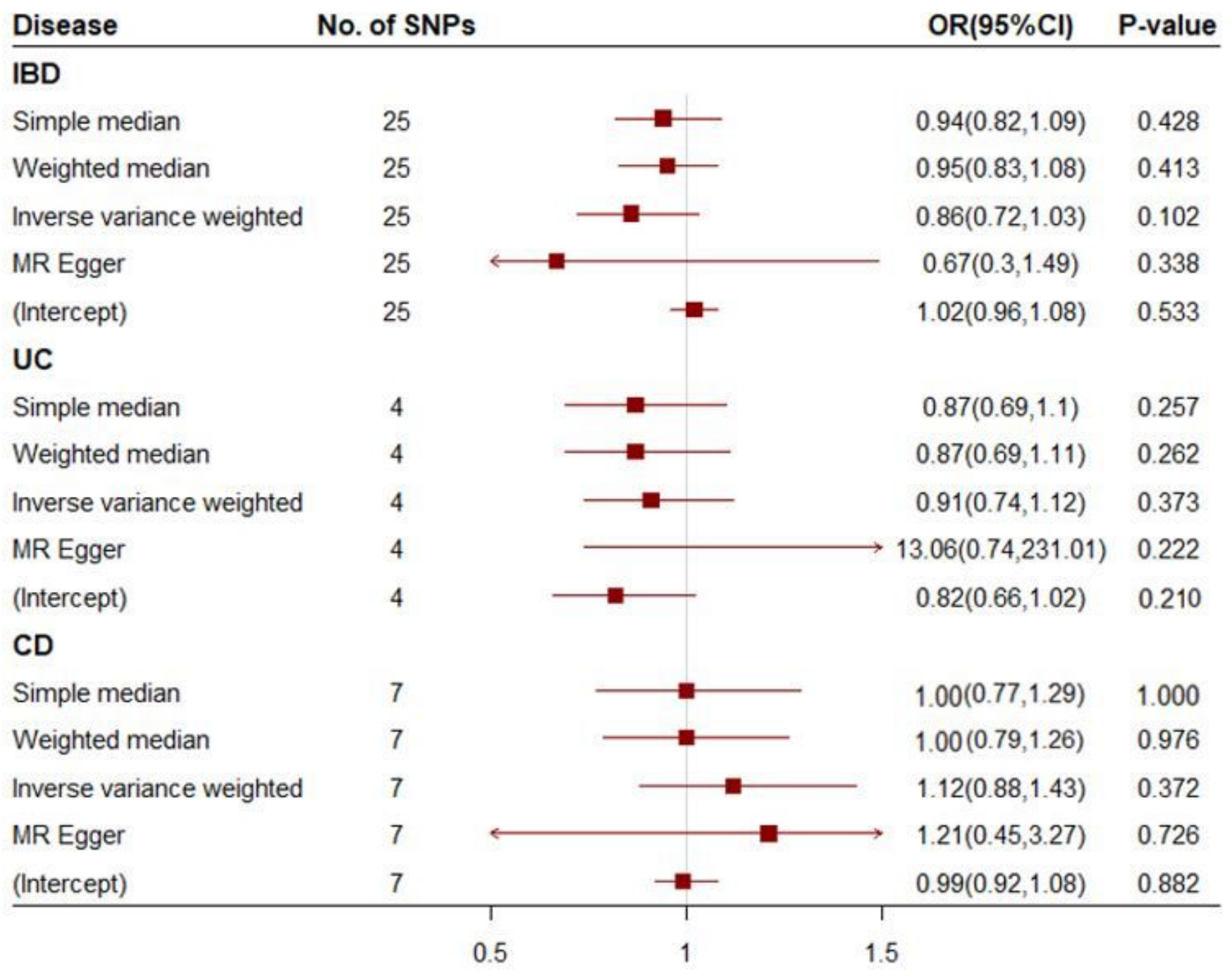

\section{Figure 3}

Odds ratio for association of genetically predicted IBD, UC and CD with RA, using four different Mendelian randomization methods. OR: odds ratio; $\mathrm{Cl}$ : confidence internal; RA, rheumatoid arthritis; IBD, inflammatory bowel disease; UC, ulcerative colitis; CD, Crohn's disease; RA, Rheumatoid Arthritis. OR (95\% $\mathrm{Cl}$ ) means risk of RA per each 1-unit higher log odds in genetically predicted IBD, UC and CD.

\section{Supplementary Files}

This is a list of supplementary files associated with this preprint. Click to download.

- SupplementalTables.xlsx

- SupplementalFigures.pptx 